\title{
Potential medicinal use of forest species of the Deciduous Seasonal Forest from Atlantic Forest Biome, South Brazil
}

\author{
Ana Paula Moreira Rovedder ${ }^{1,2 *}$, Eliara Marin Piazza ${ }^{2}$, Pedro Augusto Thomas ${ }^{2}$, Roselene \\ Marostega Felker ${ }^{2}$, Rafaela Badinelli Hummel ${ }^{2}$, Jorge Antonio de Farias ${ }^{1}$ \\ ${ }^{1}$ Universidade Federal de Santa Maria, Departamento de Ciências Florestai, Santa Maria, Rio Grande do Sul, Brasil; \\ ${ }^{2}$ Universidade Federal de Santa Maria, Núcleo de Estudos e Pesquisas em Recuperação de Áreas Degradadas, Santa \\ Maria, Rio Grande do Sul, Brasil;
}

\begin{abstract}
The current paper focuses on potential medicinal use of forest species from Deciduous Seasonal Forest in central region of Rio Grande do Sul State, South Brazil. Floristic and phytosociological surveys were carried out in 2011 and 2012. Results were compared with the available information in scientific literature about popular knowledge and biological/pharmacological evidences. Each species was classified in use categories to diseases, symptoms our disorders based on the International Statistical Classification of Diseases and Related Health Problems (ICD). In 2011, we found a total of 31 species, 77,4\% of them with medicinal use. In 2012, we found 33 species,72,7\% of them with medicinal use. The species most frequently mentioned were Casearia sylvestris, Eugenia uniflora, Cabralea canjerana, Luehea divaricata, Parapiptadenia rigida and Vitex megapotamica. The main species with biological/pharmacological properties were C. sylvestris, E. uniflora, $L$. divaricata, V. megapotamica and Zanthoxylum rhoifolium. We concluded that there is a considerable potential for medicinal purposes to forest species from Deciduous Seasonal Forest in the study region. We recommend further studies mainly about the species Prunus myrtifolia, Zanthoxylum rhoifolium, Cabralea canjerana and Casearia sylvestris -which presented references to antitumoral properties.
\end{abstract}

Key words: multiple uses, Forest Remnant, Traditional Knowledge.

*Authors for correspondence: anarovedder@gmail.com 


\section{INTRODUCTION}

Conservation of native forests requires further scientific information regarding the socioeconomic value of native species. To countries in tropical and subtropical regions it is especially importānt to find ‘sustainable use and enhancement strategies to forest remnants..Brazil is a megadiverse country, therefore, the ecosystems sustainable management is fundamental to its development. Atlantic Forest biome has covered $15 \%$ of the Brazilian territory. Nowadays the biome presents a decrease of $88 \%$ in its original area. Even so, it still holds $33-36 \%$ of the Brazilian biodiversity and is a major global biodiversity hotspot (Campanili and Schaeffer 2010). The majority of forest remnants are within the rural properties (Mariot and Reis 2010) and, usually, farmers think that areas are obstacles to its economic production and do not realize the resources and services provided by forests.

The current debate about forest conservation has focused on the hypothesis that sustainable use is more efficient to native germplasm conservation than total exclusion of human society. Successful conservation strategies with sustainable economic use requires the rescue of traditional ecological knowledge in order to find information about potential uses and management adapted to local conditions and stimulate new researches in various fields of science. Among these ones, the potential medicinal of plant species is an poorly explored theme.

The high diversity of Atlantic Forest biome shows great potential for medicinal purposes, and since exploited sustainably, can be important to social development (Pedroso et al. 2007; Campanili and Schaeffer 2010). Popular medicine based on traditional ecological knowledge is widely used for treating pain and diseases in Brazilian rural and urban areas (Maciel et al. 2002). Plants have attracted scientific interest in the search for new substances to treat many pathologies. However, the traditional ecological knowledge and most of the relevant literature are about herbaceous species; there is a lack of knowledge about medicinal potential of forest species.. In southern Brazil, Deciduous Seasonal Forest is a vegetation type of Atlantic Forest biome that is still little known and presents a high degree of habitat fragmentation. There is a precarious rescue of ethnobotanical knowledge in this region and existing studies are incipient. Some studies show that the main medicinal plants used in the region are exotic herbs from Europe, Asia or other regions of American continent (Dorigoni et al. 2001; Vendruscolo and Mentz 2006). Furthermore, knowledge about native forest species is harmed by replacing the forest cover to agriculture and pasture. The present study aimed to gather information about the potential medicinal use of native forest species of Deciduous Seasonal Forest in the Central Region of Rio Grande do Sul State, South Brazil, to colaborating with strategies and polices conservation.

\section{MATERIAL AND METHODS}

Study area - The experiment was carried out in Deciduous Seasonal Forest remnant at the "State Park Fourth Colony" conservation unit, located on Fourth Colony of Italian Immigration, Central Region of Rio Grande do Sul (RS), south Brazil, coordinates $29^{\circ} 37^{\prime} 40.80^{\prime \prime} \mathrm{S}$ and $53^{\circ} 22^{\prime \prime} 00: 38$ "'O. According to Köppen' climate classification, central region of Rio Grande do Sul has a humid subtropical climate (Cfa), without dry season and with 1300-1800 $\mathrm{mm} \mathrm{yr}^{-1}$ average annual rainfall (Moreno 1961).

The region was colonized by Italian immigrants from the year 1887 and later by German immigrants, and his descendants compose the actual population. The agrarian structure is based on small and middle sized family farms small and medium family properties and its main commercial crops are tobacco, potato and rice. The immigrant families growth increased the substitution from native forests to food crops. After deflation in rural population in the last decades, because of the Green Revolution and due to urbanization increase it is observed some forest regeneration, mainly in the slopes. On the other hand, riparian forests in the plains are still reduced.

Sampling and data collection - The survey was performed in selected área in 2011 and was repeated in the same plots in 2012.

In this area were established 16 fixed plots of 10 $\mathrm{x} 10 \mathrm{~m}$ where all individuals of forest species with circumference at breast height $(\mathrm{CBH})$ greater than or equal to $15 \mathrm{~cm}$ (class II: $\mathrm{CBH} \geq$ 
$15 \mathrm{~cm}$ ) were identified. These plots were subdivided into 64 subplots with $5 \times 5 \mathrm{~m}$ each where all individuals with circumference at breast height between 5.1 to 14.9 (Class I: $\mathrm{CBH}$ $\geq 5.1 \leq 14.9 \mathrm{~cm})$ were identified. The sample units were distributed in a stratified and randomized way in the study area.

Data Analysis - Every forest individuals in the two forest class intervals (I and II) were identified to species level, according to Angiosperm Phylogeny Group III (APG III 2012). It was calculated the density (AD), frequency (AF) and dominance (DoA), in absolute values, as well as the importance value index (IVI), according to Mueller-Dambois and Ellemberg (1974).

Bibliographical records of medicinal uses for forest species identified in floristic-phytosociological survey were collected and separated into two classes: uses in popular medicine and biological/pharmacological studies. The species were classified according to their categories of treatment use to diseases, symptoms or desorders on International Statistical Classification of Diseases and Related Health Problems (ICD) (WHO 2013): (endocrine, nutritional and metabolic diseases; diseases of the eye and adnexa; diseases of the ear and mastoid process; diseases/symptons of the circulatory system; mental and behavioural disorders; diseases of the nervous system; diseases of the respiratory system; diseases of the digestive system; diseases of the skin and subcutaneous tissue; diseases of the genitourinary system; diseases of the musculoskeletal system and connective tissue; injury, poisoning and certain other consequences of external causes; symptoms, signs and abnormal clinical and laboratory findings, not elsewhere classified; external causes of morbidity and mortality; others).

\section{RESULTS AND DISCUSSION}

Floristic and ecological characterization - In the floristic-phytosociological survey of 2011, 1,046 individuals were identified and distributed in 31 species and 16 botanical families. From these ones, there were reports about their medicinal use in 24 species, rate of $77.4 \%$. In the class interval I $(5.1 \geq \mathrm{CBH} \leq$

$14.9 \mathrm{~cm}$ ) there were 24 native forest species, being 19 the ones which present medicinal potential. In the class interval II $(\mathrm{CBH} \geq 15 \mathrm{~cm})$, there were 23 native forest species, 21 ones with medicinal potential. In the second assessment, taken in 2012, 930 individuals were identified, distributed in 33 species and 17 botanical families, with reported medicinal potential to 24 species $(72.7 \%)$. In the class interval I $(5.1 \geq \mathrm{CBH} \leq 14.9 \mathrm{~cm}), 29$ species were identified, 20 ones with medicinal properties. In the class interval II $(\mathrm{CBH} \geq 15 \mathrm{~cm}), 27$ species were identified and 22 with medicinal potential. Comparing both surveys, it was verified reduction in the total number of individuals from the year 2011 to 2012. This result may be due to the initial stage of succession, with short cycle species gradually replaced by shade tolerant species. In the second survey (2012), three species with medicinal potential were found, but that did not appear in 2011: Cedrela fissilis (Class II), Cordia aAmericana (Class II) and Apuleia leiocarpa (Class I) (Table 1 and Table 2).

The main botanical families with medicinal potential in both surveys were Sapindaceae, Salicaceae, Euphorbiaceae and Myrtaceae. Chaves and Manfredi (2010) highlighted Euphorbiaceae and Myrtaceae as being families with greater abundance of medicinal forest species in south Brazil.

Allophylus edulis, Casearia sylvestris and Sebastiania commersoniana stood out with the highest values of AD, AF, ADo and IVI in 2011 and 2012, in I and II classes (Table 1 and Table 2). These species are pointed out as ones of the most important ones according to the phytosociological point of view for the Deciduous Seasonal Forest (Longhi et al. 2000). Budke et al. (2004) found higher values of $\mathrm{AD}$ and $\mathrm{AF}$ for $S$. commersoniana. Longhi et al. (1999) and Longhi et al. (2000) found higher IVI for $C$. sylvestris. These studies were performed in the Peripheral Depression, sedimentary basin in central region of Rio Grande do Sul, same physiographic region of the present study.

Some medicinal species were restricted to a sampling class: Apuleia leiocarpa and Vitex megapotamica were present only in class I $(5.1 \geq$ $\mathrm{CBH} \leq 14.9 \mathrm{~cm})$ (Table 1). Erythroxylum deciduum, Ocotea pulchella, Prunus myrtifolia, Trichilia claussenii and Zanthoxylum rhoifolium were found only in class II (CBH $\geq 15 \mathrm{~cm}$ ) (Table 2). Presence or absence of species in the sampling classes might indicate a constant transformation in forest remnant, including mortality and recruitment, and these might be related to herbivory and competition. Another hypothesis is the natural 
evolution of forest remnant that may restrict the development of species that demand more light and, on the other hand, make that tolerant species settle. The microclimate changes that occur as the succession processes probably allow that a greater number of species settle down.

Recordings medicinal potential - C. sylvestris, $E$. uniflora, V. megapotamica, $Z$. rhoifolium, $L$. divaricata and $C$. canjerana stood out by the total number of medicinal use indications (Table 3). $C$. sylvestris and $E$. uniflora are the only native forest species found in this research that are in the National list of Medicinal Plants for the Unique Brazilian Health System (RENISUS). Most species cited in RENISUS are exotic, which is indicative of the traditional knowledge lack, problem linked to historical deforestation, predatory extraction and consequent local cultures discharacterization.. Ethnobotanical studies have shown the exotic species predominance (Dorigoni et al. 2001). Mentz et al. (1997) present s medicinal species catalogued by D'Ávila in 1910 and used in Rio Grande do Sul state. From 296 species, 210 are native from Rio Grande do Sul state. Among these 55 are arboreous species It is noticed that a larger amount of information is necessary to understand forest species of regional ecosystems. . However, to researches on medicinal potential, studies carried out in the forest remnant are necessary, once the habitat fragmentation* ${ }^{*}$ have reduced the available biodiversity.

Additionally, the low insertion of native species in RENISUS list reflects the lack of incentives for research on this topic in the Brazilian scientific scenario. There is a gap in public policy, financial resources and funding for research on native species.

From these indications in popular medicine, it is pointed out the use against cancer and tumors related for Casearia sylvestris, Luehea divaricata, Matayba eleagnoides, Myrsine umbellata, Ocotea puberula and Cordia americana (Longhi 1995; Franco and Barros 2006; Rodrigues and Carvalho 2001; Santos et al. 2010; Ferreira et al. 2011).

Records of adverse effects also must be considered. C. fissilis, Sapium glandulatum and $S$. romanzoffiana could cause vomiting, skin irritation and diarrhea, respectively (Valle and Kaplan 2000; Franco and Barros 2006). Reports indicates that stems and roots decoction of $C$. canjerana is narcotic and abortive causes vomiting and, depending on the dose, it can even causes death (Klein, 1984)

Table 1. Absolute density (AD), Absolute frequency (AF), Absolute dominance (ADo) and importance value index (IVI) for species with medicinal use found in the floristic-phytosociological survey in interval class $\mathrm{I}(5.1 \geq \mathrm{CBH}$ $\leq 14.9 \mathrm{~cm}$ ), in the years 2011 and 2012 .

\begin{tabular}{|c|c|c|c|c|c|c|c|c|c|}
\hline \multicolumn{2}{|c|}{$\begin{array}{l}\text { Interval Class I } \\
(5.1 \geq \mathrm{CBH} \leq 14.9 \mathrm{~cm})\end{array}$} & \multicolumn{4}{|l|}{2011} & \multicolumn{4}{|l|}{2012} \\
\hline Species & $\begin{array}{l}\text { Botanical } \\
\text { Family }\end{array}$ & $\mathrm{AD}$ & $\mathrm{AF}$ & Ado & IVI & $\mathrm{AD}$ & $\mathrm{AF}$ & Ado & IVI \\
\hline $\begin{array}{l}\text { Allophylus } \\
\text { edulis }\end{array}$ & Sapindaceae & 287.50 & 56.25 & 1.49 & 27.25 & 243.75 & 56.25 & 1.33 & 27.38 \\
\hline $\begin{array}{l}\text { Apuleia } \\
\text { leiocarpa }\end{array}$ & Fabaceae & $*$ & $*$ & $*$ & $*$ & 12.50 & 12.50 & 0.46 & 5.18 \\
\hline $\begin{array}{l}\text { Cabralea } \\
\text { canjerana }\end{array}$ & Meliaceae & 6.25 & 6.25 & 0.05 & 1.51 & 6.25 & 6.25 & 0.06 & 1.61 \\
\hline $\begin{array}{l}\text { Casearia } \\
\text { sylvestris }\end{array}$ & Salicaceae & 181.25 & 37.50 & 1.49 & 22.79 & 193.75 & 37.50 & 0.93 & 19.90 \\
\hline $\begin{array}{l}\text { Cedrela } \\
\text { fissilis }\end{array}$ & Meliaceae & 6.25 & 6.25 & 0.14 & 2.13 & 18.75 & 18.75 & 0.23 & 5.03 \\
\hline $\begin{array}{l}\text { Cupania } \\
\text { vernalis }\end{array}$ & Sapindaceae & 12.50 & 12.50 & 0.02 & 2.53 & 12.50 & 12.50 & 0.03 & 2.64 \\
\hline $\begin{array}{l}\text { Eugenia } \\
\text { uniflora }\end{array}$ & Myrtaceae & 81.25 & 43.75 & 0.35 & 12.17 & 43.75 & 31.25 & 0.34 & 8.70 \\
\hline $\begin{array}{l}\text { Jacaranda } \\
\text { micrantha }\end{array}$ & Bignoniaceae & 100.00 & 50.00 & 0.89 & 17.39 & 100.00 & 50.00 & 1.12 & 18.73 \\
\hline
\end{tabular}




\begin{tabular}{|c|c|c|c|c|c|c|c|c|c|}
\hline $\begin{array}{l}\text { Luehea } \\
\text { divaricata }\end{array}$ & Malvaceae & 25.00 & 18.75 & 0.19 & 5.10 & 25.00 & 18.75 & 0.26 & 5.50 \\
\hline $\begin{array}{l}\text { Matayba } \\
\text { eleagnoides }\end{array}$ & Sapindaceae & 93.75 & 25.00 & 0.85 & 13.13 & 87.50 & 25.00 & 1.30 & 15.50 \\
\hline $\begin{array}{l}\text { Myrsine } \\
\text { umbellata }\end{array}$ & Primulaceae & 50.00 & 31.25 & 0.14 & 7.68 & 56.25 & 37.50 & 0.18 & 9.24 \\
\hline $\begin{array}{l}\text { Nectandra } \\
\text { megapotanica }\end{array}$ & Lauraceae & 68.75 & 25.00 & 0.59 & 10.44 & 75.00 & 25.00 & 0.76 & 11.67 \\
\hline $\begin{array}{l}\text { Ocotea } \\
\text { puberula }\end{array}$ & Lauraceae & 68.75 & 25.00 & 0.59 & 10.44 & 75.00 & 25.00 & 0.76 & 11.67 \\
\hline $\begin{array}{l}\text { Parapiptadeni } \\
\text { a rigida }\end{array}$ & Fabaceae & 31.25 & 25.00 & 0.67 & 9.42 & 18.75 & 12.50 & 0.16 & 3.69 \\
\hline $\begin{array}{l}\text { Patagonula } \\
\text { Cordia }\end{array}$ & Boraginaceae & 18.75 & 12.50 & 0.16 & 3.73 & 25.00 & 12.50 & 0.18 & 4.11 \\
\hline $\begin{array}{l}\text { Sapium } \\
\text { glandulatum }\end{array}$ & Euphorbiaceae & 100.00 & 43.75 & 0.53 & 14.11 & 93.75 & 43.75 & 0.61 & 14.48 \\
\hline $\begin{array}{l}\text { Sebastiana } \\
\text { commersonian } \\
\text { a }\end{array}$ & Euphorbiaceae & 131.25 & 37.50 & 0.81 & 16.28 & 112.50 & 37.50 & 0.85 & 15.78 \\
\hline $\begin{array}{l}\text { Syagrus } \\
\text { romanzoffiana }\end{array}$ & Arecaceae & 68.75 & 31.25 & 1.36 & 16.32 & 87.50 & 31.25 & 1.95 & 20.35 \\
\hline $\begin{array}{l}\text { Trema } \\
\text { micrantha }\end{array}$ & Cannabaceae & 18.75 & 18.75 & 0.09 & 4.21 & 18.75 & 18.75 & 0.10 & 4.29 \\
\hline $\begin{array}{l}\text { Vitex } \\
\text { megapotamica }\end{array}$ & Lamiaceae & 6.25 & 6.25 & 0.01 & 1.30 & 6.25 & 6.25 & 0.003 & 1.41 \\
\hline
\end{tabular}

Table 2. Absolute density (AD), Absolute frequency (AF), Absolute dominance (ADo) and importance value index (IVI) for species with medicinal use found in the floristic-phytosociological survey in interval class II $(\mathrm{CBH} \geq 15 \mathrm{~cm})$, in the years 2011 and 2012.

\begin{tabular}{|c|c|c|c|c|c|c|c|c|c|}
\hline $\begin{array}{l}\text { Interval Class } \\
\text { II } \\
(\mathrm{CBH} \geq 15 \\
\mathrm{cm})\end{array}$ & 2011 & & & & & 2012 & & & \\
\hline Species & $\begin{array}{l}\text { Botanical } \\
\text { Family }\end{array}$ & $\mathrm{AD}$ & $\mathrm{AF}$ & Ado & IVI & $\mathrm{AD}$ & $\mathrm{AF}$ & ADo & IVI \\
\hline $\begin{array}{l}\text { Allophylus } \\
\text { edulis }\end{array}$ & Sapindaceae & 793.75 & 53.12 & 1.08 & 61.39 & 868.75 & 57.81 & 3.15 & 56.52 \\
\hline $\begin{array}{l}\text { Cabralea } \\
\text { canjerana }\end{array}$ & Meliaceae & 50.00 & 7.81 & 0.02 & 3.81 & 43.75 & 6.25 & 0.27 & 4.35 \\
\hline $\begin{array}{l}\text { Casearia } \\
\text { sylvestris }\end{array}$ & Salicaceae & 287.50 & 32.81 & 0.11 & 17.64 & 237.50 & 32.81 & 1.23 & 21.82 \\
\hline $\begin{array}{l}\text { Cedrela } \\
\text { fissilis }\end{array}$ & Meliaceae & $*$ & $*$ & $*$ & $*$ & 6.25 & 1.56 & 0.01 & 0.65 \\
\hline $\begin{array}{l}\text { Cupania } \\
\text { vernalis }\end{array}$ & Sapindaceae & 25.00 & 3.25 & 0.01 & 1.71 & 12.50 & 3.12 & 0.02 & 1.30 \\
\hline $\begin{array}{l}\text { Erythroxylum } \\
\text { deciduum }\end{array}$ & $\begin{array}{l}\text { Erythroxylace } \\
\text { ae }\end{array}$ & 6.25 & 1.56 & 0.009 & 0.77 & $*$ & * & * & $*$ \\
\hline $\begin{array}{l}\text { Eugenia } \\
\text { uniflora }\end{array}$ & Myrtaceae & 312.50 & 35.93 & 0.22 & 22.19 & 325.00 & 31.25 & 2.15 & 29.00 \\
\hline
\end{tabular}




\begin{tabular}{|c|c|c|c|c|c|c|c|c|c|}
\hline $\begin{array}{l}\text { Jacaranda } \\
\text { micrantha }\end{array}$ & Bignoniaceae & 50.00 & 10.93 & 0.004 & 4.95 & 25.00 & 4.68 & 0.14 & 2.72 \\
\hline $\begin{array}{l}\text { Luehea } \\
\text { divaricata }\end{array}$ & Malvaceae & 37.50 & 7.81 & 0.02 & 3.27 & 12.50 & 3.12 & 0.03 & 1.33 \\
\hline $\begin{array}{l}\text { Matayba } \\
\text { eleagnoides }\end{array}$ & Sapindaceae & 87.50 & 15.62 & 0.07 & 7.89 & 81.25 & 12.50 & 0.48 & 8.16 \\
\hline $\begin{array}{l}\text { Myrsine } \\
\text { umbellata }\end{array}$ & Primulaceae & 218.75 & 34.37 & 0.12 & 16.76 & 231.25 & 32.81 & 0.95 & 20.12 \\
\hline $\begin{array}{l}\text { Nectandra } \\
\text { megapotanica }\end{array}$ & Lauraceae & 43.75 & 7.81 & 0.03 & 3.78 & 37.50 & 6.25 & 0.24 & 3.98 \\
\hline $\begin{array}{l}\text { Ocotea } \\
\text { puberula }\end{array}$ & Lauraceae & 18.75 & 4.68 & 0.01 & 1.95 & 6.25 & 1.56 & 0.03 & 0.78 \\
\hline $\begin{array}{l}\text { Ocotea } \\
\text { pulchella }\end{array}$ & Lauraceae & 17.50 & 10.93 & 0.12 & 10.22 & 68.75 & 6.25 & 0.42 & 5.85 \\
\hline $\begin{array}{l}\text { Parapiptadeni } \\
\text { a rigida }\end{array}$ & Fabaceae & 6.25 & 1.56 & 0.006 & 0.68 & 6.25 & 1.56 & 0.06 & 0.93 \\
\hline $\begin{array}{l}\text { Cordia } \\
\text { americana }\end{array}$ & Boraginaceae & $*$ & $*$ & $*$ & $*$ & 6.25 & 1.56 & 0.03 & 0.76 \\
\hline $\begin{array}{l}\text { Prunus } \\
\text { myrtifolia }\end{array}$ & Rosaceae & 12.50 & 18.75 & 0.07 & 9.53 & 81.25 & 12.50 & 0.55 & 8.52 \\
\hline $\begin{array}{l}\text { Sapium } \\
\text { glandulatum }\end{array}$ & Euphorbiaceae & 68.75 & 14.06 & 0.07 & 6.91 & 31.25 & 6.25 & 0.24 & 3.79 \\
\hline $\begin{array}{l}\text { Sebastiana } \\
\text { commersonian } \\
a\end{array}$ & Euphorbiaceae & 168.75 & 20.31 & 0.12 & 12.14 & 118.75 & 17.18 & 0.59 & 10.96 \\
\hline $\begin{array}{l}\text { Syagrus } \\
\text { romanzoffiana }\end{array}$ & Arecaceae & 12.50 & 3.12 & 0.01 & 1.55 & 6.25 & 1.56 & 0.07 & 1.01 \\
\hline $\begin{array}{l}\text { Trema } \\
\text { micrantha }\end{array}$ & Cannabaceae & 50.00 & 10.93 & 0.04 & 4.95 & 6.25 & 1.56 & 0.09 & 1.10 \\
\hline $\begin{array}{l}\text { Trichilia } \\
\text { claussenii }\end{array}$ & Meliaceae & 18.75 & 3.12 & 0.01 & 1.59 & 6.25 & 1.56 & 0.01 & 0.67 \\
\hline $\begin{array}{l}\text { Zanthoxylum } \\
\text { rhoifolium }\end{array}$ & Rutaceae & 12.50 & 3.12 & 0.009 & 1.28 & 12.50 & 3.12 & 0.09 & 1.64 \\
\hline
\end{tabular}

* Species absent in interval class or sampling survey period.

Table 3. Medicinal use indication to forest species of Deciduous Seasonal Forest based on International Statistical Classification of Diseases and Related Health Problems (ICD), Atlantic Forest biome, Rio Grande do Sul State, South Brazil.

\begin{tabular}{|c|c|c|}
\hline $\begin{array}{l}\text { Disease/ Sympton } \\
\text { /disorders Group }\end{array}$ & Species & Medicinal use \\
\hline $\begin{array}{l}\text { Endocrine, nutritional and } \\
\text { metabolic diseases. }\end{array}$ & 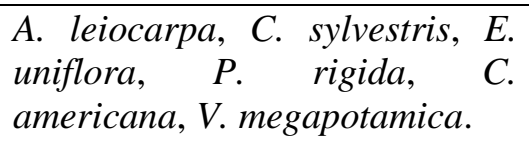 & $\begin{array}{l}\text { Rickets, diabetes. Regulating } \\
\text { levels of triglycerides, } \\
\text { cholesterol and uric acid. }\end{array}$ \\
\hline $\begin{array}{l}\text { Diseases of the eye and } \\
\text { adnexa. }\end{array}$ & T. micrantha. & Eye pain. \\
\hline $\begin{array}{l}\text { Diseases of the ear and } \\
\text { mastoid process. }\end{array}$ & Z. rhoifolium. & Earache. \\
\hline $\begin{array}{l}\text { Diseases/ symptons of the } \\
\text { circulatory system. }\end{array}$ & $\begin{array}{l}\text { A. edulis, C. sylvestris, C. fissilis, } \\
\text { E. uniflora, L. divaricata, } M .\end{array}$ & $\begin{array}{l}\text { Hypertension, necrotic lesions, } \\
\text { stroke, cardiovascular disease, }\end{array}$ \\
\hline
\end{tabular}




\begin{tabular}{|c|c|c|}
\hline & $\begin{array}{l}\text { eleagnoides, } P . \quad \text { rigida, } \quad V . \\
\text { megapotamica. }\end{array}$ & $\begin{array}{l}\text { hemorrhoids, circulation, } \\
\text { inflammation of the lymph } \\
\text { nodes. }\end{array}$ \\
\hline $\begin{array}{l}\text { Mental and behavioural } \\
\text { disorders. }\end{array}$ & C. fissilis, L. divaricata. & Stuttering, sleep regulation. \\
\hline $\begin{array}{l}\text { Diseases of the nervous } \\
\text { system. }\end{array}$ & $\begin{array}{l}\text { C. canjerana, } C \text {. sylvestris e } E \text {. } \\
\text { uniflora. }\end{array}$ & $\begin{array}{l}\text { Bacterial meningitis } \\
\text { meningitis in or meningitis } \\
\text { due to other and unspecified } \\
\text { causes. } \\
\text { Soothing. }\end{array}$ \\
\hline
\end{tabular}

Diseases of the respiratory A. edulis, C. vernalis, E. uniflora, Asthma, sinusitis, bronchosystem.

$J$. micrantha, $L$. divaricata, $P$. pulmonary disorders and rigida, $P$. myrtifolia, $V$. catarrhal, airway clearance, megapotamica. $\quad$ sore throat.

Diseases of the digestive A. edulis, C. canjerana, C. Treatment of digestive system.

sylvestris, C. fissilis, E. problems (diarrhea,
deciduum, E. uniflora, $L$. constipation, dyspepsia, ulcers, divaricata, M. eleagnoides, M. gastritis), oral aphthae, umbellata, Ocotea. pulchella, $C$. gingivitis, toothache, liver americana, $P$. rigida, $S$. ailments. romanzoffiana, $T$. claussenii, $V$. megapotamica, Z. rhoifolium.

Diseases of the skin and subcutaneous tissue.

A. edulis, C. canjerana, C. Eczema, itch, cuts, boils, sylvestris, $\quad C$. fissilis, $J$. dermatosis, cancerous wounds. micrantha, $L$. divaricata, $M$. umbellata, N. megapotamica, $O$. puberula, $P$. rigida, $C$. americana, T. micrantha, $V$. megapotamica.

Diseases of the C. canjerana, C. sylvestris, C. Arthritis, rheumatism, musculoskeletal system fissilis, E. uniflora, J. micrantha, arthralgias, fractures, muscle and connective tissue. L. divaricata, M. eleagnoides, $N$. megapotamica, $\quad P$. rigida, $\quad T$. bruises, gout, neuralgia. micrantha, V. megapotamica.

Diseases of the $C$. canjerana, $C$. sylvestris, $C$. Inflammation of the testes, genitourinary system. fissilis, E. uniflora, L. divaricata, renal and urinary disorders, $O$. pulchella, $P$. rigida, $S$. leucorrhea, inflammation of commersoniana,

S.romanzoffiana, $V$. megapotamica.

Injury, poisoning and C. sylvestris. Burns. certain other consequences of external causes. 


\begin{tabular}{|c|c|c|}
\hline $\begin{array}{lll}\text { Symptoms, signs } & \text { and } \\
\text { abnormal clinical } & \text { and } \\
\text { laboratory findings, not } \\
\text { elsewhere classified. }\end{array}$ & $\begin{array}{l}\text { A. edulis, } C . \text { canjerana, } C . \\
\text { sylvestris, C. fissilis, E. uniflora, } \\
\text { M. eleagnoides, C. americana, } V . \\
\text { megapotamica, Z. rhoifolium. }\end{array}$ & $\begin{array}{l}\text { Jaundice, edemas, vomiting, } \\
\text { hemorrhaging, } \\
\text { headache, throes, halitosis, } \\
\text { intestinal colic. }\end{array}$ \\
\hline $\begin{array}{l}\text { External causes of } \\
\text { morbidity and mortality. }\end{array}$ & $\begin{array}{l}\text { A. leiocarpa, C. sylvestris, } C . \\
\text { fissilis, M. umbellata, Sapium } \\
\text { glandulatum, Z. rhoifolium. }\end{array}$ & Snake bite and insect bites. \\
\hline Others. & $\begin{array}{l}\text { A. leiocarpa, C. canjerana, } C . \\
\text { sylvestris, C. fissilis, C. vernalis, } \\
\text { E. uniflora, J. micrantha, } L . \\
\text { divaricata, M. eleagnoides, } O . \\
\text { puberula, C. americana, } P . \\
\text { myrtifolia, S. gladulatum, } T . \\
\text { micrantha, T. claussenii, } V . \\
\text { megapotamica, Z. rhoifolium. }\end{array}$ & $\begin{array}{l}\text { Anxiety, Astringent, emetic, } \\
\text { tonic, stimulant, diaphoretic, } \\
\text { analgesic and anti- } \\
\text { inflammatory (for unspecified } \\
\text { body site), antioxidant, } \\
\text { depurative and depurative of } \\
\text { blood. }\end{array}$ \\
\hline
\end{tabular}

However, species listed in popular medicine could not ensure a successful use. Studies to prove the medicinal properties of these species are still insufficient. To some species identified in the Table 4. Biological/pharmacological activities for forest species found in Deciduous Seasonal Forest, Atlantic Forest Biome, Brazil.

\begin{tabular}{ll}
\hline Species & Biological Activities \\
\hline Allophylus edulis & Anticholinesterasic and antioxidant activity (Umeo et al. 2011). \\
Apuleia leiocarpa & A ntidiabetic activity (Carvalho 2003), antiophidian activity \\
Cabralea canjerana & (Ruppelt et al. 1991). \\
Casearia sylvestris & Antitumor and antifungal activity (Cardoso-Lopes et al. 2008). \\
& Antihypoglycemic (Werle et al. 2009), antitumor (Felipe et al. \\
& 2014, Santos et al. 2010), antimicrobial activity (Basile et al. \\
& 1990), anti-ulcer activity (Basile et al. 1990; Sertié et al. 2000), \\
anti-inflammatory activity (Esteves et al., 2005) antiophidian \\
Cedrela fissilis & activity (Raslan et al. 2002). \\
Eugenia uniflora & Antibacterial activity (Cortez et al. 1998). \\
& antimicrobial (Brun and Mossi 2010; Castro et al. 2010; \\
& Auricchio et al. 2007), antioxidant activity (Auricchio et al. \\
Luehea divaricata & 2007) \\
Nectandra megapotamica & Antiulcerogenic activity (Bighetti et al. 2004). \\
Prunus myrtifolia & antimicrobial (Garcez et al. 2009), antioxidant activity (Garcez \\
& et al. 2009). \\
Vitex megapotamica & Cytotoxic, antifungal, antioxidant e antitumor activity (Santos et \\
Zanthoxylum rhoifolium & al. 2005). \\
& Hypolipidemic activity (Brandt et al. 2009). \\
& Antimicrobial activity (Gonzaga et al. 2003), antitumor activity \\
& (Silva et al. 2007)
\end{tabular}

C. canjerana, C. sylvestris, P. myrtifolia and $Z$. rhoifolium distinguished themselves for presenting anti-tumor activity. (Felipe et al. 2014; Santos et al. 2005; Silva et al. 2007). On the order hand, a same species. On the order hand, a same species may current paper, promising results were found to prove their biological/pharmacological activities (Table 4). 
damage using C. canjerana, C. fissilis, S. gladulatum and $S$. romanzoffiana (Klein, 1984; Cooke 1931; Valle and Kaplan 2000; Carvalho 2006) show this necessity, because they have beneficial effects too. Such studies may avoid risks to the population. Absence of public policies, investments and incentives to research delay the scientific development about medicinal potential of Brazilian species. Fortunately, since the early 2000s, the Brazilian Health Ministry has developed programs and actions for the sector. Efforts to insert medicinal plants in the Brazilian Public Health System (SUS) has been performed, such as the Medicinal Plants National Policy (2001), the National Seminary of Medicinal Plants, Herbal Medicine and Pharmaceutical Care (2003) and the National Proposal Inter-Working Group of medications and medicinal plants (Rodrigues et al. 2006).In law n. 11.428 from December 22nd 2006, that is about the usage and protection for Atlantic Forest vegetation, forest sub-products extractivism is allowed since the species are not at risk and the specific legal limitations are observed (Brazil 2006). It is noticed that legislation makes it possible planting and sustainable exploration of Atlantic Forest species. The species researched in this paper are options for these initiatives in order to value the preserved areas. However, according to Reis and Mariot (2002), too much extraction for obtaining medicinal plants has led to population reduction for some species. Thus, sustainable strategies for planting and handling are fundamental for medicinal species conservation and make them available for consumption. According to World Health Organization (WHO) around 25\% of modern medicine comes originally from plants used in a traditional way (WHO 2002). It is also necessary researches about the sustainable crop and handling for these species and their habitats in order to protect them. However, to success these recommendations, it is necessary to create mechanisms to stimulate rural communities so that they can interact again with the remaining natural forests through the development of market for nontimber products, including medicinal plants.

On the other hand, disorganization or absence of productive chains for non-timber products leads to an underground market, to incorrect use and, consequently, to environmental and economical sustainability lack. There is the necessity for public policies formulation to organizing local productive arrangements for medicinal plants, making it possible for the communities to know more about planting, processing and marketing the products obtained from forest conservation. C-consequences of this process would be social, economical and environmental benefits once this economic diversification, mainly in small rural farms, empowers them through the development of new products from their regions as well as native germplasm and ethnobotanical knowledge conservation.

Concerning to native forest species for medicinal use, there is a wide variety of plants that have been unexplored because of the public policies absence to rescuing the ethno-knowledge of indigenous cultures and traditional rural communities.

\section{CONCLUSION}

Species diversity of Deciduous Seasonal Forest holds potentials for the medicinal use that not been explored yet and can be alternative sources of income. Many reported forest species have wide use in popular medicine. However, there is a need for pharmacological and toxicological researches to prove their potential and to avoid risks to human population. The species Casearia sylvestris and Eugenia uniflora deserve special attention in future scientific studies about their pharmacological activities due to the great number of reported uses. Although the forest remnant presents initial ecological succession, it showed a considerable number of species with potential medicinal use. This result shows the importance of conservation to natural ecosystems, even with habitat fragmentation, stimulating local succession to express the full potential to alternative uses.

Once the ethnobotanical knowledge, in many Brazil' regions, is suppressed or little studied, floristic research is useful to highlight the medicinal potential of native species. The public policies formulation for development and implementation of local productive arrangements is a necessary strategy to forest conservation with participation of local communities.

\section{ACKNOWLEDGEMENTS}

The authors thank the National Council of Scientific and Technological Development (CNPq) and the State Environment Secretary of Rio Grande do Sul state (SEMA). 


\section{REFERENCES}

Angiosperm Phylogeny Group. An update of the Angiosperm Phylogeny Group classification for the orden and families of flowering plants: APG III. 2012.

Auricchio MT, Bugno A, Barros SBM, Bacchi EM. Antimicrobial and antioxidant activities and toxicity of Eugenia uniflora. Lat Am J Pharm. 2007; 26 (1): 76-81.

Basile AC, Sertié JA, Panizza S, Oshiro TT, Azzolini CAJ. Pharmacological assay of Casearia sylvestris. I: Preventive anti-ulcer activity and toxicity of the leaf crude extract. J Ethnopharmacol. 1990; 30(2): 185-97.

Bighetti, A. E.; Antônio, M. A.; Possenti, Ana; Foglio, M. A.; Siqueira,.G.; Carvalho, J.E. Efeitos da administração aguda e subcrônica da Luehea divaricata Martus et Zuccarini. Lecta 2004; 22 (1/2): 53-58. Brandt AP, Oliveira LFS, Fernandes FB, Alba J. Avaliação in vivo do efeito hipocolesterolêmico e toxicológico preliminar do extrato bruto hidroalcoólico e decocção da Vitex megapotamica (Spreng) Moldenke (V. montevidensis Cham.). Rev Bras Farmacognosia. 2009; 19(2): 388-393.

Brasil. Presidência da República, Lei Federal 11428 [internet]. Brasília: 2006. [updated in: 09/01/2007; Acess in: 25/05/ 2013]. Available in: http://www.planalto.gov.br/ccivil_03/_ato20042006/2006/lei/111428.htm.

Brito MR, Senna-Valle L. Plantas medicinais utilizadas na comunidade caiçara da Praia do Sono, Paraty, Rio de Janeiro, Brasil. Rio de Janeiro, Brazil. Acta Bot. Bras. 2011; 25(2): 363-372.

Brun GR, Mossi AJ. Caracterização química e atividade antimicrobiana do óleo volátil de pitanga (Eugenia uniflora L.). Perspec. 2010; 34(127): 135-142.

Budke JC, Giehl ELH, Athayde EA, Eisinger SM, Záchia RA. Florística e fitossociologia do componente arbóreo de uma floresta ribeirinha, arroio Passo das Tropas, Santa Maria, RS, Brasil. Acta Bot. Bras. 2004; 18(3): 581-589.

Campanili M, Schaffer WB. Mata Atlântica: patrimônio nacional brasileiro. 2. ed. Brasília: MMA; 2010. 408 p.

Cardoso-Lopes EMC, Rosana C, Carreira RC, Agripino DG, Torres LMB, Cordeiro I, et al. Screening for antifungal, DNA-damaging and anticholinesterasic activities of Brazilian plants from the Atlantic Rainforest - Ilha do Cardoso State Park. Rev. Bras. Farmacogn. 2008; 18 (Supl.): 655-660.

Carvalho PER. Espécies Florestais Brasileiras. 1 ed. Brasília: Embrapa; 2003. 1039 p. Carvalho PER. Espécies Florestais Brasileiras. 2 ed. Brasília: Embrapa; 2006. 627 p.

Castro RD, Freires IA, Ferreira DAH, Jovito VC, Paulo MQ Atividade antibacteriana in vitro de produtos naturais sobre Lactobacillus casei . Int J Dent. 2010; 9(2): 74-77.

Chaves CL, Manfredi CS. Árbóreas medicinais das matas ciliares do Rio Canoas: potencialidades de uso em projetos de restauração. Rev. Bras. Plantas Med. 2010; 12 (3): 322-332.

Corrêa MP. Dicionário de plantas úteis do Brasil e exóticas cultivadas. 2 ed. Rio de Janeiro: Minist. Agric.; 1931. 707 p.

Cortez DAG, Cortez LER, Nakamura TU, Nakamura CV. Antibacterian activity of extracts from wood of

Cedrela fissilis Vell. (Meliaceae). Acta Sci. 1998; 20(2): 243-245.

Dorigoni PA, Ghedini PC, Fróes LF, Baptista KC, Ethur ABM, Baldisseroto B, et al. Levantamento de dados sobre plantas medicinais de uso popular no município de São João do Polêsine, RS-I - Relação entre enfermidades e espécies utilizadas. Rev. Bras. Plantas Med. 2001; 4(1): 69-79.

Felipe KB, Kviecinski MR, da Silva FO, Bücker NF, Farias MS, Castro LS. et al. Inhibition of tumor proliferation associated with cell cycle arrest caused by extract and fraction from Casearia sylvestris (Salicaceae). J Ethnopharmacol. 2014; 155(3): 1492-1499.

Ferreira PM, Costa-Lotufo LV, Moraes MO, Barros FWA, Martins AMA, Cavalheiro AJ, et al. Folk uses and pharmacological properties of Casearia sylvestris: a medicinal review. An Acad. Bras. Cienc. 2011; 83(4): 1373-1384.

Esteves I, Souza IR, Rodrigues M, Cardoso LGVL, Santos S, Sertié JAA, et al. Gastric antiulcer and antiinflammatory activities of the essential oil from Casearia sylvestris Sw. J Ethnopharmacol. 2005; 101: 191-196.

Franco EAP, Barros RFM. Uso e diversidade de plantas medicinais no Quilombo Olho D’água dos Pires, Esperantina, Piauí. Rev. Bras. Plantas Med. 2006; 8(3): $78-88$.

Garcez FR, Garcez WS, Hamerski L, Miguita CH. Fenilpropanóides e outros constituintes bioativos de Nectandra megapotamica. Quím. Nova. 2009; 32(2): 407-411.

Gonzaga W, Weber AD, Giacomelli SR, Ionara ID, Hoelzel SCS, Morel AF. Antibacterial Alkaloids from Zanthoxylum rhoifolium. Planta Med. 2003; 69(4): 371-374.

Klein RM. Meliáceas. In: Reitz, R. Flora Ilustrada de Santa Catarina. Itajaí: Itajaí; 1984. 138 p. Longhi R A. Livro de árvores: árvores e arvoretas do Sul. 2. ed. Porto Alegre: L\&PM; 1995. 176 p.

Longhi SJ, Araujo MM, Kelling MB, Hoppe JM, Müller I, Borsoi GA. Aspectos fitossociológicos de fragmento de Floresta estacional Decidual, Santa Maria, RS. Ci. Fl. 2000; 10 (2): 59-74.

Longhi SJ, Nascimento ART, Fleig FD, Della-Flora JB, Freitas RA, Charão LW. Composição florística e estrutura da comunidade arbórea de um fragmento 
florestal no município de Santa Maria - Brasil. Ci. Fl. 1999; 9 (1): 115-133.

Maciel MAM, Pinto AC, Veiga Jr, VF. Plantas medicinais: a necessidade de estudos multidisciplinares.

Quím. Nova. 2002; 25(3): 429-438.

Mariot A, Reis MS. Biodiversidade e sua importância como fonte de plantas medicinais. Rev. Ci. Agrovet. 2006; 5(1): 60-69.

Mentz LA, Lutzemberger LC, Schenkel EP. Flora medicinal do Rio Grande do Sul: notas sobre a obra de D'ávila (1910). Cad. Farm. 1997; 13(1):25-48.

Moreno JA. Clima do Rio Grande do Sul. 1 ed. Porto Alegre: Secr. Agric.; 1961. 42 p.

Mueller-Dombois D, Ellenberg H. Aims and methods of vegetation ecology. 1 ed. New York: Wiley; 1974. $547 \mathrm{p}$.

Pedroso K, Watzlawick LF, Oliveira, NK, Valério AF, Gomes GS, Silvestre R. Levantamento de plantas medicinais arbóreas e ocorrência em Floresta Ombrófila Mista. Amb. 2007; 3(1): 39-50.

Raslan DS, Jamal CM, Duarte DS, Borges MH, Lima ME. Anti-PLA2 action test of Casearia sylvestris

Sw. Boll Chim Farm. 2002; 141(6): 457-460.

Reis MS, Mariot A. Diversidade natural e aspectos agronômicos de plantas medicinais. In: Simões CMO, Schenkel EP, Gosmann G, Mello JCP, Mentz LA, Petrovick PR. Farmacognosia - da planta ao medicamento. 3 ed. Porto Alegre: UFRGS; 2002. p. 41-62.

Rodrigues AG, Santos MG, Amaral ACF. A política pública sobre plantas medicinais e fitoterápicos. In: Ministério da Saúde. A Fitoterapia no SUS e do Programa de Plantas Medicinais do Centro de Investigação de Drogas. 1 ed. Brasília: Minist. Saúde; 2006. p. 9-28.

Rodrigues VEG, Carvalho DA. Levantamento etnobotânico de plantas medicinais no domínio das pradarias da região do Alto Rio Grande - Minas Gerais. Ciênc Agrotec. 2001; 25(1):102 -123.

Ruppelt BM, Pereira EF, Gonçalves LC, Pereira NA. Pharmacological screening of plants recommended by folk medicine as anti-snake venom: I. Analgesic and anti-inflammatory activities. Mem Inst Oswaldo Cruz. 1991; 86(2): 203-205.

Santos AG, Ferreira PMP, Vieira-Júnior GM, Perez CC, Tininis AG, Silva GH, et al. Casearin $X$, its degradation product and other clerodane diterpenes from leaves of Casearia sylvestris: evaluation of cytotoxicity against normal and tumour human cells. Chem Biodivers. 2010; 7(1): 205-215.

Santos LA, Silva DHS, Bolzani VS. Utilização de técnicas hifenadas na identificação de derivados do ácido cafeico em folhas de Prunus myrtifolia (Rosaceae). In: $28^{\mathrm{a}}$ Reunião da Sociedade Brasileira de Química (SBQ); 2005. Poços de Caldas: Livro de Resumos, 2005. v. 28.
Sertié JAA, Carvalho JCT, Panizza S. Antiulcer Activity of the Crude Extract from the Leaves of Casearia Sylvestris. Pharm Biol. 2000; 38( 2 ): 112-119.

Silva SL, Figueiredo PM, Yano T. Avaliação citotóxica do óleo volátil extraído das folhas do

Zanthoxylum rhoifolium Lam.. Acta Amaz. 2007; 37(2): 281-286.

Umeo SH, Yokota ME, Romagnolo MB, Laverde A. Avaliação das propriedades antioxidantes, anticolinesterásicas e citotóxicas dos frutos de Allophylus edulis (a.st.-hil., Cambess. \& a. juss.) Radlk. (sapindaceae). Arq. Ciênc. Saúde. 2011; 15(2): 167-171.

Valle LS, Kaplan MAC. Sapium glandulatum complex (Euphorbiaceae). An Acad. Bras. Ciênc. 2000; 72(2): 293-294.

Vendruscolo GS, Mentz LA. Levantamento etnobotânico das plantas utilizadas como medicinais por moradores do bairro Ponta Grossa, Porto Alegre, Rio Grande do Sul, Brasil. Acta Bot. Bras. 2006; 20(2): 367-382.

Werle ALB, Zanetti GD, Ceron CS, Mafron MP. Avaliação da atividade de Casearia sylvestris Swartz (Flacourtiaceae) sobre os níveis séricos de triglicerídeos em ratos. Rev. Bras. Farmacog. 2009; 19(2): 400- 402.

World Health Organization. Launches the first global strategy on traditional and alternative medicine [internet]. 2002 [acess in: 15/09/2012]. Available in: http://

http://www.who.int/mediacentre/news/releases/relea se38/en/.

World Health Organization. International Statistical Classification of Diseases and related Health Problems [internet]. 2013 [acess in: 13/04/2013]. Available in: http://apps.who.int/classifications/icd10/browse/201 $0 /$ en. 